\title{
Instrumentos para avaliação do padrão de sono em crianças com doenças crônicas: revisão integrativa
}

\author{
Instruments for assessing sleep patterns in children with chronic diseases: an integrative review \\ Instrumentos para evaluar el patrón de sueño en niños con enfermedades crónicas: revisión integrativa
}

Rosilene Silva de Andradel; Ravini dos Santos Fernandes Vieira dos Santos"; Antônio Eduardo Vieira dos Santos"I"; Neize Lúcia de Andrade ${ }^{v}$; Isabela Fornerolli de Macedov; Michelle Darezzo Rodrigues Nunesvi.

\begin{abstract}
RESUMO
Objetivo: analisar a produção científica nacional e internacional relacionada aos instrumentos utilizados para avaliar padrão de sono em crianças com doenças crônicas. Método: revisão integrativa realizada em bases de dados (PubMed, Web of Science, CINHAL, LILACS, SciELO) que compreendeu o período de 2006-2016. Resultados: foram selecionados 16 artigos para compor a revisão e encontrados vários instrumentos confiáveis para avaliar o sono, porém nenhum adaptado para o Português do Brasil, com exceção da polissonografia e do actigraphy. Crianças com doenças crônicas apresentam distúrbios do sono que se correlacionavam com dor, fadiga, problemas de comportamento, falta de atividade física e obesidade. Conclusão: no Brasil, o uso de instrumentos específicos para avaliar o sono em crianças ainda é pouco explorado. A utilização desses instrumentos na prática clínica poderia auxiliar enfermeiros a traçarem estratégias para diminuir os distúrbios do sono na população infanto-juvenil.
\end{abstract}

Descritores: Sono; criança; adolescente; escala; enfermagem pediátrica.

\section{ABSTRACT}

Objective: to examine Brazilian and international scientific production on the use of sleep pattern assessment instruments in children with chronic diseases. Method: this integrative review was conducted on the PubMed, Web of Science, CINHAL, LILACS, and SciELO databases for 2006 to 2016. Results: 16 articles were selected for review, and several reliable sleep assessment instruments were found. However, none was adapted to Brazilian Portuguese, except for polysomnography and actigraphy. Children with chronic diseases have sleep disorders that correlate with pain, fatigue, behavior problems, lack of physical activity, and obesity. Conclusion: in Brazil, specific sleep assessment instruments are little used with children. Use of these instruments in clinical practice could help nurses to outline strategies to reduce sleep disorders among children and adolescents.

Descriptors: Sleep; child; adolescent; scale;pediatric nursing.

\section{RESUMEN}

Objetivo: analizar la producción científica nacional e internacional en cuanto a los instrumentos utilizados para evaluar el patrón de sueño en niños con enfermedades crónicas. Método: se trata de una revisión integrativa realizada en bases de datos (PubMed, Web of Science, CINHAL, LILACS, SciELO) en el período de 2006-2016. Resultados: se seleccionaron 16 artículos para componer la revisión y se encontraron varios instrumentos confiables para evaluar el sueño, pero ninguno adaptado a Portugués de Brasil, con excepción de la polisomnografía y del actigrafía. Los niños con enfermedades crónicas presentan trastornos del sueño que se asocian con dolor, cansancio, problemas de comportamiento, falta de actividad física y obesidad. Conclusión: en Brasil, el uso de instrumentos específicos para evaluar el sueño en niños todavía es poco explorado. La utilización de estos instrumentos en la práctica clínica podría ayudar a los enfermeros a trazar estrategias para disminuir los disturbios del sueño en la población infanto-juvenil.

Descriptores: Sueño; niño; adolescente; escala; enfermería pediátrica.

\section{INTRODUÇÃo}

As doenças crônicas representam $60 \%$ de todo ônus decorrente de doenças no mundo, e estima-se que este valor alcance os $80 \%$ em 2020 . No Brasil, a prevalência de doenças crônicas entre crianças de 0 a 14 anos é de $9,3 \%{ }^{1}$.
As principais características da condição crônica de saúde são o caráter permanente, a incapacidade residual, a longa duração, a dependência medicamentosa, o caráter recorrente e o fato de quase 
sempre ser incurável, irreversível e degenerativa², predispondo ao comprometimento do padrão de sono na criança.

Interrupções frequentes do sono e repouso em crianças interfere nos processos de crescimento, desenvolvimento, aprendizagem e saúde ${ }^{3,4}$. Além de impedir a função reparadora e homeostática, alterações no padrão do sono podem levar a consequências cardiovasculares, metabólico-endócrinas, imunológicas, psicológicas, aumento da sonolência diurna, diminuição na qualidade de vida ${ }^{5}$.

O tempo total de sono varia com cada faixa etária e diminui gradativamente com o passar dos anos, variando em média 12 horas no recém-nascido e até 7 a 8 horas no escolar e adolescente $e^{3,6}$. Em crianças hospitalizadas, se evidencia diminuição significativa da eficiência do sono (em média 3,4 horas) principalmente devido aos diversos despertares noturnos e cochilos diurnos ${ }^{4,7-9}$.

Todo cuidado que priorize a manutenção do sono e repouso favorecerá a cura, pois criará condições propícias para o organismo combater a doença, impedindo sua exacerbação e desencadeamento de novos distúrbios $^{6}$. A enfermagem pode planejar e atuar com eficácia na diminuição dos distúrbios do sono em crianças com doenças crônicas e hospitalizadas mediante utilização de instrumentos para mensurar o padrão de sono.

O objetivo deste estudo foi analisar a produção científica nacional e internacional relacionada aos instrumentos utilizados para avaliar padrão de sono em crianças com doenças crônicas.

\section{Metodologia}

Trata-se de revisão integrativa realizada nas seguintes etapas: formulação do problema; procedimentos para busca; avaliação dos dados; análise dos dados; e interpretação e apresentação da revisão ${ }^{10,11}$. A pergunta norteadora foi: Quais são os instrumentos utilizados para avaliar o padrão de sono em crianças com doença crônica?

Para responder a essa pergunta, as bases de dados pesquisadas foram: PUBMED; Web of Science e CINHAL; LILACS e SciELO. Utilizaram-se os seguintes descritores em Ciência da Saúde (DeCS) e Medical Subject Headings (MESH): sono (sleep), criança (children), adolescente (teen), escala (scale); e as seguintes palavras-chaves: padrão de sono (sleep pattern), instrumento (instrument), dispositivo (device) - tanto os descritores quanto as palavras-chave foram usados em diferentes combinações.

Foram incluídos artigos originais, publicados entre os anos de 2006 e 2016, nas línguas inglesa ou portuguesa. Foram excluídos artigos de revisão e dissertações/teses.

Os dados foram organizados em duas categorias relacionadas à exploração dos seus conteúdos. Inicialmente, foram encontrados 303 artigos. Após leitura exaustiva dos resumos, foram excluídos todos os materiais que se encontravam em duplicidade, bem como os que não pertenciam à temática do estudo. Assim, restaram 16 artigos $^{12-27}$ para serem analisados em maior profundidade. O fluxograma da seleção dos estudos para a revisão integrativa é representado na Figura 1.

\section{RESULTADOS}

A maioria dos estudos foi publicada nos Estados Unidos (68,7\%), e o período de publicação variou entre 2006 e 2016 , com maior concentração $(68,7 \%)$ nos anos de 2014 (37,5\%), 2015 (18,7\%) e 2016 (12,5\%). Em relação ao tipo de periódico, $6(37,5 \%)$ foram publicados em revistas científicas não pertencentes à área de enfermagem, dos quais 4 (25\%), em periódicos de especialidades que abordam desordens mentais; 2 (12,5\%), em periódicos de especialidades pediátricas. Os outros 10 $(62,5 \%)$ artigos foram publicados em revistas científicas de enfermagem, dos quais $6(37,5 \%)$, em periódicos de enfermagem em oncologia pediátrica; 3 (18,7\%), em periódicos de enfermagem pediátrica; e 1 (6,2\%), em periódico de pesquisa básica em enfermagem.

A análise dos 16 estudos selecionados ${ }^{12-27}$ permitiu realizar a síntese quantitativa a qual evidenciou, além dos instrumentos que avaliam o sono, outros utilizados para relacionar demais sintomas, tais como dor, fadiga, ansiedade, depressão, estresse, comportamento, alimentação, satisfação dos pais e fatores ambientais. Os instrumentos usados para avaliar o sono - questionários e escalas - encontrados nos estudos selecionados e sua respectiva descrição estão descritos na Figura 2.

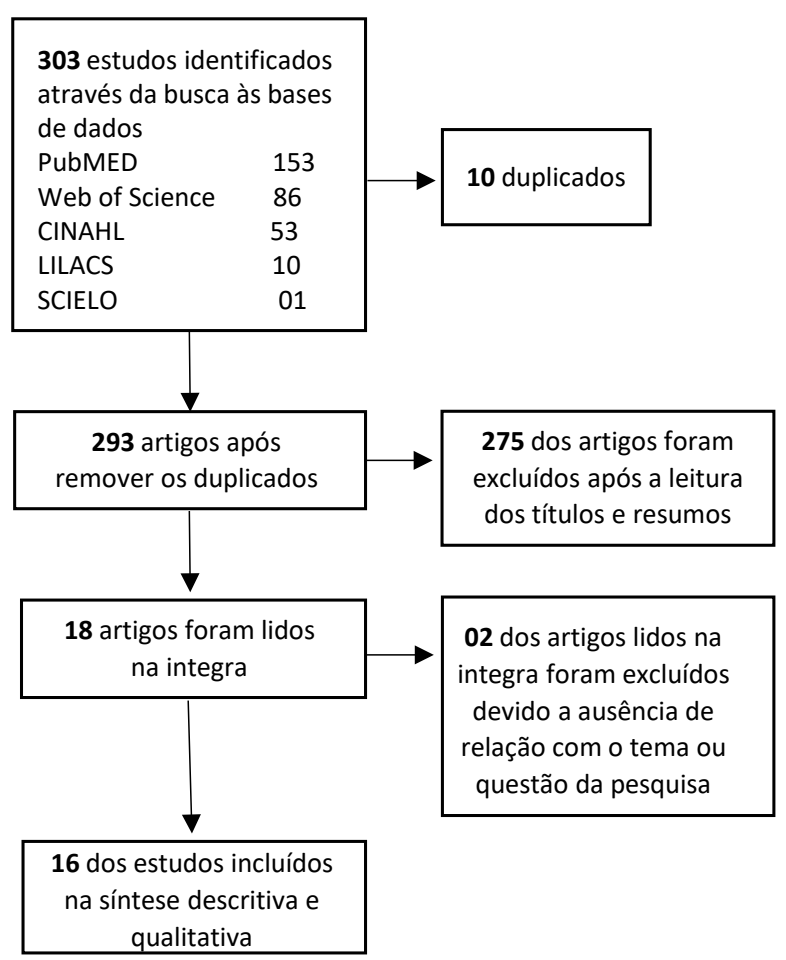

FIGURA 1: Fluxograma de seleção dos estudos para a revisão integrativa. Rio de Janeiro, 2017. 
Os instrumentos que avaliam o sono-diários e dispositivos - são apresentados na Figura 3.

Os 16 estudos selecionados foram agrupados em duas categorias, que são analisadas a seguir ${ }^{12-27}$.

\section{Categoria 1: Avaliação do sono em crianças com doen- ças crônicas}

Quatro estudos compõem esta categoria, que avalia exclusivamente o sono em crianças com doenças crônicas.

O primeiro estudo avaliou o sono em 51 adolescentes (10-19 anos) com diagnóstico de câncer. Os instrumentos utilizados foram respondidos pelos adolescentes. Os resultados revelaram piora na qualidade e higiene do sono de adolescentes em tratamento quimioterápico ${ }^{12}$.

O segundo estudo analisou o sono em 107 crianças (1-18 anos) hospitalizadas. Os pais completaram o diário para as crianças menores de 12 anos; após essa idade, a própria criança/adolescente respondeu ao instrumento. O estudo apresentou média de 9,8 horas de sono, variando entre cinco e 16 horas. Crianças de 2 e 5 anos foram as mais afetadas, $52,3 \%$ das crianças tiveram um sono pobre, 49,5\% tiveram uma eficiência de sono inferior a $90 \%$ e $34,5 \%$ acordaram três ou mais vezes. A maior prevalência do mau sono ocorreu em crianças com condições crônicas (61,9\%). O despertar do sono estava relacionado aos cuidados de enfermagem, alarmes e dor ${ }^{13}$.

O terceiro estudo analisou o sono em 95 crianças e adolescentes (3-17 anos) com diagnóstico de neurofibromatose. $O$ instrumento para avaliação do sono foi realizado pelos pais. $\mathrm{O}$ estudo indicou que essas crianças demoram a iniciar o sono, apresentando distúrbios de manutenção do sono, transição do sono-vigília e sonolência diurna ${ }^{14}$.

O quarto estudo avaliou o efeito de uma intervenção no sono de 22 crianças (5-15 anos) com diagnóstico de autismo/deficiência intelectual. A intervenção utilizada foi um treinamento dos pais com relação à abordagem comportamental da criança. Os instrumentos utilizados para avaliar o sono foram realizados pelos pais. O estudo revelou que o sono da criança foi melhorando se comparado ao período pré-intervenção. Observou-se uma diminuição nos despertares noturnos e melhora na resistência a dormir ${ }^{15}$.

Os estudos analisados em seus resultados concordam que crianças e adolescentes portadores de condições crônicas apresentam um sono prejudicado. Entre os diversos fatores relacionados, está o tratamento realizado, a hospitalização, a presença de sintomas relacionados à doença e as interrupções do sono geradas pelo ambiente e cuidados da equipe de Enfermagem.

NOME DO INTRUMENTO

DESCRIÇÃO

BISQ Brief Infant Sleep

Questionnaire (um)

CSHQ Children's Sleep Habits

Questionnaire (quarto)

PSQI Índice de qualidade de

Pittsburg (um)

CSHQ-A Children's Sleep Habits

Questionnaire-Abbreviated

(um)

Sleep Routines "Before You

Were Sick" Questionnaire (um)

TSBS-BISQ Toddler Sleep

Behavior Scale (um)

SDSC Sleep Disturbance Scale for Children (um)

GSDS General Sleep

Distuburbance Scale (um) -

NRS Numeric Ratin Scale (dois)

Adolescent Sleep Wake Scale (Sleep Quality) (um)

\section{QUESTIONÁRIOS}

Questionário de sono infantil com 11 perguntas sobre o padrão de sono diurno e comportamento noturno, baseado no sono da última semana.

Questionário dos hábitos do sono infantil, avalia como foi o tipo do sono nas últimas semanas.

Questionário com 19 itens relacionados a qualidade do sono e perturbações do sono no mês anterior.

Questionário de 22 itens para crianças de 4 a 12 anos, projetadas para medir os hábitos de sono e perturbações do sono na semana passada. Índices mais altos indicam maior distúrbio de sono.

Breve questionário usado para avaliar os padrões basais de sono (durante a semana e final de semana). É um relatório retrospectivo sobre o sono antes do início dos sintomas relacionados ao diagnóstico de câncer.

\section{ESCALAS}

Avalia o comportamento do sono de bebês, inclui as noites acordado, tempo de início e o tempo acordado antes de adormecer, local onde dormiu (quarto dos pais ou não), local aonde dorme (cama ou não) e problemas com o sono. Maior pontuação representa melhores comportamentos do sono.

Escala que classifica os distúrbios do sono em seis categorias: distúrbio da iniciação e manutenção do sono, distúrbios respiratórios do sono, de excitação/pesadelos, de transição dormir-despertar, da sonolência diurna excessiva e hiperidrose.

Escala composta por 21 itens que avalia à dificuldade de dormir, despertar noturno, acordar muito cedo, qualidade e quantidade de sono, fadiga e alerta no trabalho e uso de substâncias para ajudar a induzir o sono no mês passado.

Escala de 0 a 10 que avalia a quantidade de sono com as seguintes perguntas: Quanto de sono você teve durante a noite?, Quanta atividade você teve hoje?

Escala que avalia a qualidade do sono de adolescentes de 12 a 18 anos através de 28 itens, e 5 dimensões comportamentais: indo para cama, adormecer, manter o sono, reiniciar o sono e despertar. As pontuações mais altas indicam melhor qualidade do sono.

FIGURA 2: Descrição dos instrumentos que avaliam sono: questionários e escalas. Rio de Janeiro, 2017. 


\section{NOME DO INTRUMENTO}

\section{BISQ Brief Infant Sleep}

Questionnaire (um)

CSHQ Children's Sleep Habits

Questionnaire (quarto)

PSQI Índice de qualidade de

Pittsburg (um)

CSHQ-A Children's Sleep Habits

Questionnaire-Abbreviated

(um)

Sleep Routines "Before You

Were Sick" Questionnaire (um)

\section{TSBS-BISQ Toddler Sleep}

Behavior Scale (um)

SDSC Sleep Disturbance Scale for Children (um)

\section{GSDS General Sleep}

Distuburbance Scale (um) -

NRS Numeric Ratin Scale (dois)

Adolescent Sleep Wake Scale (Sleep Quality) (um)

\section{DESCRIÇÃO}

\section{QUESTIONÁRIOS}

Questionário de sono infantil com 11 perguntas sobre o padrão de sono diurno e comportamento noturno, baseado no sono da última semana.

Questionário dos hábitos do sono infantil, avalia como foi o tipo do sono nas últimas semanas.

Questionário com 19 itens relacionados a qualidade do sono e perturbações do sono no mês anterior.

Questionário de 22 itens para crianças de 4 a 12 anos, projetadas para medir os hábitos de sono e perturbações do sono na semana passada. Índices mais altos indicam maior distúrbio de sono.

Breve questionário usado para avaliar os padrões basais de sono (durante a semana e final de semana). É um relatório retrospectivo sobre o sono antes do início dos sintomas relacionados ao diagnóstico de câncer.

\section{ESCALAS}

Avalia o comportamento do sono de bebês, inclui as noites acordado, tempo de início e o tempo acordado antes de adormecer, local onde dormiu (quarto dos pais ou não), local aonde dorme (cama ou não) e problemas com o sono. Maior pontuação representa melhores comportamentos do sono.

Escala que classifica os distúrbios do sono em seis categorias: distúrbio da iniciação e manutenção do sono, distúrbios respiratórios do sono, de excitação/pesadelos, de transição dormir-despertar, da sonolência diurna excessiva e hiperidrose.

Escala composta por 21 itens que avalia à dificuldade de dormir, despertar noturno, acordar muito cedo, qualidade e quantidade de sono, fadiga e alerta no trabalho e uso de substâncias para ajudar a induzir o sono no mês passado.

Escala de 0 a 10 que avalia a quantidade de sono com as seguintes perguntas: Quanto de sono você teve durante a noite?, Quanta atividade você teve hoje?

Escala que avalia a qualidade do sono de adolescentes de 12 a 18 anos através de 28 itens, e 5 dimensões comportamentais: indo para cama, adormecer, manter o sono, reiniciar o sono e despertar. As pontuações mais altas indicam melhor qualidade do sono.

FIGURA 3: Descrição dos instrumentos que avaliam sono: diários e dispositivos. Rio de Janeiro, 2017.

\section{Categoria 2: Avaliação do sono e sua correlação com outros sintomas}

Desta categoria emergiram quatro subcategorias, englobando 12 estudos ${ }^{16-27}$. Eles abordam o sono e sintomas como dor, fadiga, problemas de comportamento e os relacionam com o sono de crianças com doenças crônicas. Esta categoria foi desmembrada em quatro subcategorias conforme os sintomas estudados.

\section{Subcategoria 1: Alterações do sono e problemas de comportamento}

Essa subcategoria é composta por três estudos sobre sono e problemas de comportamento em crianças com doença crônica ${ }^{16-18}$.

O primeiro estudo ${ }^{16}$ comparou a qualidade do sono e os problemas comportamentais em 102 crianças (6-12 anos) com diagnóstico de asma. $\mathrm{O}$ instrumento utilizado foi a polissonografia, realizada nas crianças; e o Child Behavior Checklist (CBCL), respondido pelas crianças e pelos pais. $\mathrm{O}$ estudo relata que as crianças com asma demoram mais para adormecer e apresentam maior movimentação nas pernas durante o sono, assim como maior sono REM e menor proporção do sono de onda lenta, apresentando maior índice de excitação respiratória ${ }^{16}$.
O segundo estudo também compara os distúrbios do sono e problemas de comportamento (ansiedade, depressão, dificuldades de ajuste do humor) em 116 pacientes (6-11 anos) com diagnóstico de artrite idiopática juvenil. $\mathrm{O}$ instrumento de sono utilizado foi relatado pelos pais. Foi utilizado o Child Behavior Checklist ( $\mathrm{CBCL}$ ) e o Scale Pain, ambos relatados pelos pais. O estudo evidenciou que crianças com artrite idiopática juvenil apresentam os seguintes distúrbios do sono: resistência para dormir, atraso no início do sono, alterações na duração do sono, ansiedade, despertares noturnos, parassômnias, respiração desordenada e sonolência diurna ${ }^{17}$.

O terceiro estudo avaliou o sono da mãe e da criança (3-12 anos) em 26 participantes com diagnóstico de leucemia linfoblástica aguda (LLA). Os instrumentos utilizados foram: Actiwatch 2, Daily Sleep e Children's Sleep Habits Questionnaire-Abbreviated (CSHQ-A). Foram utilizados outros instrumentos para avaliar a ansiedade e a depressão. $O$ estudo expõe que mães e crianças com LLA têm distúrbio do sono, as crianças com LLA demoram mais tempo para adormecer e tiveram variações no padrão do sono. Identificou-se correlação entre a insônia da mãe, estresse, depressão e ansiedade ${ }^{18}$. 
Crianças com doenças crônicas, apresentam distúrbios do sono que podem contribuir para o desenvolvimento de problemas comportamentais na criança e na mãe.

\section{Subcategoria 2: Sono e fadiga}

O primeiro estudo analisou o sono e a fadiga em 29 participantes (7-18 anos) com diagnóstico de câncer. Os instrumentos que avaliam o sono foram utilizados nas crianças; e o Daily Sleep, respondido pelos pais. Os instrumentos que avaliam a fadiga foram respondidos pelas crianças, exceto o Fatigue Scale-parent (FS-P), que foi respondido pelos pais. 0 estudo relata que $70 \%$ das crianças dormiram de 1 a 2 horas sem despertar, $22 \%$ dormiram 2 horas/noite e $7 \%$ das crianças dormiram 4 horas de sono ininterruptos. O número de despertar noturno está relacionado à fadiga ao longo da hospitalização, e os dados do Actigraph mostraram que há distúrbio do sono ${ }^{19}$.

O segundo estudo avaliou o sono e a fadiga em 77 crianças e adolescentes (4-18 anos) com diagnóstico de leucemia linfoblástica aguda. O instrumento utilizado para avaliar o sono foi o Children's Sleep Habits Questionnarie (CSHQ). As crianças menores de 13 anos tiveram ajuda dos pais para preencherem o questionário, e as com idade superior a 13 anos completaram-no sozinhas. Além do instrumento do sono, foram utilizados instrumentos que avaliam fadiga e o General Sleep Disturbance Scale (GSDS), que foram preenchidos pelos pais. O estudo apresenta que crianças em tratamento quimioterápico dormem menos do que o recomendado, apresentam perturbações no sono; as crianças de 8 a 12 anos relatam fadiga; e a fadiga e distúrbios do sono foram significativamente correlacionados ${ }^{20}$.

O terceiro estudo avaliou sono e fadiga em 35 crianças e adolescentes (8-17 anos) com diagnóstico de câncer. Os participantes foram recrutados no hospital e utilizaram os instrumentos em casa. Foram usados o Actigraph e a PedsQL Escala Multidimensional do Cansaço. $\mathrm{O}$ estudo evidenciou que crianças e adolescentes com câncer tem problemas com sono e fadiga mesmo em casa. Também revelou que os adolescentes dormiram menos que as crianças e ficaram mais horas acordados após o início do sono. As crianças e adolescentes que tiveram menor duração do sono foram também os que tiveram mais problemas com a fadiga ${ }^{21}$.

\section{Subcategoria 3: Sono e dor}

O primeiro estudo analisou o sono e a dor em 27 crianças (5-19 anos) com diagnóstico de anemia falciforme. O estudo apresentou que $50 \%$ dos episódios de dor ocorrem no primeiro dia de internação e que mais de $50 \%$ das crianças relatam interrupções no sono noturno, enquanto $20 \%$ delas relatam dormir durante o dia ${ }^{22}$.

O segundo estudo analisou o sono e a dor em 49 crianças (8-17 anos) com câncer e demonstrou que $22,4 \%$ das crianças apresentaram dor leve, 20,4\% dor moderada e $12,2 \%$ dor intensa. A maior classificação de dor ocorreu no primeiro dia de internação. Não houve diferença significativa do sono em relação à dor, porém a dor interferiu no sono ${ }^{23}$.

O terceiro estudo avaliou o sono, a dor e as interferências do ambiente em 15 crianças em idade escolar e com diagnóstico de câncer. Utilizou-se a escala de faces Wong-Baker ou escala numérica e HOBO $\mathrm{H}^{\mathrm{N}} \mathrm{12-012}$. $\mathrm{O}$ estudo evidenciou que as crianças com idade escolar não apresentaram um ciclo de sono completo. Os níveis sonoros noturnos, a intensidade da luz e a temperatura excederam o recomendado, interferindo no sono saudável ${ }^{24}$.

O quarto estudo dessa categoria avaliou sono e dor em 66 crianças (10-17 anos) com diagnóstico de anemia falciforme. Utilizou-se o Índice de qualidade do sono de Pittsburg (PSQI), Visual analog scale e Pediatric Pain Coping Questionnaire (PCQ). O estudo revela que dois terços das crianças relataram dor, a maioria $(91,2 \%)$ apresentou distúrbio do sono leve a grave e cerca de $18,2 \%$ utilizam medicações para dormir, três ou mais vezes por semana ${ }^{25}$.

\section{Subcategoria 4: Outras correlações do sono}

Esta subcategoria é composta por dois estudos que encontraram correlações do padrão de sono diferentes dos estudos anteriores.

O primeiro avalia ao mesmo tempo o sono, a fadiga e a dor em 40 crianças (8-14 anos) com diagnóstico de artrite idiopática juvenil. Concluiu-se que $68 \%$ das crianças com artrite idiopática juvenil tem um sono perturbado, as crianças mais novas (8-11 anos) dormiram mais e mais cedo que as crianças mais velhas (12-14 anos) ${ }^{26}$.

O segundo buscou associação entre sono e obesidade em 304 crianças (12-32 meses) nascidas a termo com mais de 2.500 g. O estudo revelou que a duração do sono noturno variou de acordo com a raça, situação socioeconômica e realização de atividade. Bebês brancos, com condição socioeconômica acima do limiar da pobreza e que realizaram mais atividade física tiveram maior duração do sono. Encontrou-se também associação entre obesidade e menor duração do sono, sendo que as crianças com menos peso dormiram 0,7 hora a mais/noite em comparação com crianças obesas ${ }^{27}$. Observou-se que a dor e a fadiga alteram o sono e que alguns fatores, como idade e excesso de peso, podem influenciar negativamente o padrão de sono das crianças.

\section{DISCUSSÃO}

Os resultados encontraram diversos instrumentos utilizados para avaliar o padrão de sono em crianças com doenças crônicas: cinco questionários, cinco escalas, dois diários do sono e três dispositivos. Quase todos os artigos citaram a validade e confiabilidade do instrumento escolhido para o estudo, porém nenhum deles foi traduzido, adaptado e validado para a língua portuguesa, com exceção dos dispositivos polissonografia e actigraphy. Para que possam ser usados cientificamente, esses instrumentos devem ser válidos e confiáveis, 
sendo necessário passar por todos os processos que garantam a viabilidade de utilização dos mesmos ${ }^{28,29}$.

Os resultados indicaram que crianças em situações crônicas apresentam distúrbios do sono, como piora na qualidade e higiene do sono e retardo do adormecimento. Quando hospitalizadas, elas dormem menos devido à própria doença e seus sintomas, ao tratamento, aos ruídos e aos cuidados da equipe de enfermagem. Estudos com neonatos e crianças com doenças crônicas demonstraram resultados semelhantes: uma revisão sobre sono de neonatos em unidade de terapia intensiva afirma que o ambiente hospitalar pode ser um facilitador do sono interrompido, devido à tecnologia disponibilizada, à baixa temperatura, à luminosidade e aos ruídos excessivos ${ }^{30}$; enquanto outro estudo, com 11 crianças (2-12 anos) hospitalizadas com câncer, demonstrou que estas tinham dificuldade para adormecer, pior qualidade do sono e dificuldades para adormecer mesmo após a alta8 .

A análise dos estudos selecionados nesta pesquisa revelou também que os distúrbios do sono podem estar relacionados com outros sintomas, como problemas comportamentais, dor e fadiga. Um estudo norueguês com crianças (11-13 anos) apresentando doença crônica demonstrou que elas tiveram níveis superiores de transtornos emocionais e comportamentais ${ }^{31}$. Outro estudo, com 83 mães e crianças (2-5 anos), apontou reatividade emocional, ansiedade/depressão, queixas somáticas, retraimento/depressão, problemas de atenção e comportamento agressivo mais alto nas crianças que apresentavam problemas de sono comparadas às que não apresentavam, enfatizando que os problemas de sono ou sono insuficiente exacerbam as dificuldades emocionais e comportamentais ${ }^{32}$. Achados de pesquisa com crianças com câncer mostraram que elas tinham mais problemas com sono, depressão, ansiedade e fadiga crônica ${ }^{33}$.

Sobre sono e fadiga, esta revisão demonstrou correlação entre os dois sintomas e também com a dor. Crianças com menor duração do sono tiveram mais problemas com fadiga e dor. Um estudo de caso-controle, com 167 participantes com câncer e 170 saudáveis, revelou que os distúrbios do sono e fadiga estavam relacionados com sofrimento psicológico, podendo desencadear a depressão ${ }^{34}$.

Em relação à correlação entre sono e dor, os resultados demonstraram que, quanto maior os sintomas de dor, maiores são as dificuldades relacionadas ao sono e menor é o número de horas de sono da criança. Estudo com 112 portadores de artrite reumatoide adultos relata que menos de $80 \%$ dos pacientes apresentaram uma má qualidade do sono, estando o mesmo relacionado à apneia do sono e depressão, assim como a dor nas articulações que está correlacionada com os distúrbios do sono, interferindo na qualidade do sono e de vida desses pacientes ${ }^{35}$. Em pesquisa com 89 mulheres com diagnóstico de fibromialgia: 76\% apresentaram um sono não reparador, não obtiveram uma boa qualidade de sono (intensificando a dor e fadiga) e manifestaram muitas das vezes problemas psíquicos, como ansiedade e depressão ${ }^{36}$.

Também se identificou que a obesidade pode ser relacionada com distúrbios do sono. Crianças obesas dormem menos que crianças saudáveis, e a atividade física pode colaborar com um sono melhor. Em um estudo com 4.231 crianças (1-4 anos) saudáveis, aquelas que dormiram por menos de 10 horas/noite tiveram grande probabilidade de apresentar excesso de peso ou obesidade aos $4 \operatorname{anos}^{37}$. Pesquisa sobre aptidão física com 200 escolares revelou que os que dormem menos de 9 horas/dia também tiveram maior taxa de índice de massa corporal. Além disso, o melhor desempenho com relação à aptidão física deu-se para o grupo de escolares que dorme mais de 9 horas/dia ${ }^{38}$.

\section{CONCLUSÃo}

Este estudo revisou 16 artigos científicos publicados entre os anos de 2006 e 2016 e relacionados aos distúrbios do sono em crianças com doenças crônicas. A maioria dessa produção foi realizada nos EUA, nos últimos quatro anos do referido período.

A maioria dos instrumentos utilizados para verificar o sono é apenas válida e confiáveis para a língua estrangeira, demonstrando uma importante lacuna para o Brasil, onde somente a polissonografia e o actigraphy já foram utilizados em estudos anteriores.

Observou-se que a doença crônica interfere negativamente no sono, cujas alterações podem levar a outros problemas, como dor, fadiga, ansiedade e ganho de peso.

Espera-se com este estudo sensibilizar a equipe de enfermagem para a relevância da melhoria da qualidade de sono diário da criança. Trata-se de assunto extremamente importante para a qualidade de vida da criança e pouco explorado na literatura nacional.

\section{REFERÊNCIAS}

1. Marcon SS, Radovanovic CAT, Waidman MAP, Olireira MLF, Sales CA. Vivência e reflexões de um grupo de estudos junto às famílias que enfrentam a situação crônica de saúde. Texto Contexto enferm. 2005; 14(spe):116-24.

2. Freitas M C, Mendes MMR. Condição crônica: análise do conceito no contexto da saúde do adulto. Rev. latinoam. enferm. (online). 2007; 15(4):590-97.

3. Souza RP, Viviani AG. Qualidade do sono em crianças hospitalizadas. Pediatr. Mod. 2014; 50(3).

4. Lee S, Narendran G, Madsen LT, Schulte F. A systematic review of sleep in hospitalized pediatric câncer patients. Psycho-oncology. 2016; 26(8):1059-69.

5. Pinto JPF. Estudo da qualidade do sono dos pacientes internados no serviço de cirurgia e de medicina interna do Centro Hospitalar Cova da Beira [tese de doutorado]. Covinhã (Pt): Universidade da Beira do Interior; 2014.

6. Paiva MB, Souza CAC, Soares E. Fatores que interferem na preservação do sono e repouso de crianças em terapia intensiva. Esc. Anna Nery Rev. Enferm. 2006; 10(1):29-35.

7. Nunes MDR. Avaliação da fadiga em crianças e adolescentes 
hospitalizados com câncer e sua relação com padrão de sono e qualidade de vida relacionada à saúde [tese de doutorado]. Ribeirão Preto (SP): Universidade de São Paulo; 2014.

8. Setoyama A, Ikeda M, Kamibeppu K. Objective assessment of sleep status and its correlates in hospitalized children with cancer: Exploratory study. Official Journal of the Japan Pediatrics International. Pediatr. int. 2016; 58(9):842-49.

9. Linder LA, Christian BJ. Nighttime sleep characteristics of hospitalized school-age children with câncer. Oncol. Nurs. forum. 2012; 39(6):553-61.

10. Whittemore R, Knafl K. The integrative review: updated methodology. J. adv. nurs. 2005; 52(5):546-53.

11. Mendes KDS, Silveira RCCP, Galvão CM. Revisão integrativa: método de pesquisa para a incorporação de evidências na saúde e na enfermagem. Texto \& contexto enferm. 2008; 17(4):758-64. 12. Walker AJ, Johnson KP, Miaskowski C, Lee KA, Gedaly-Duff. Sleep quality and sleep hygiene behaviors of adolescents during chemotherapy. J. clin. sleep med. 2010; 6(5):439-44.

13. Herbert AR, Lima J, Fitzgerald DA, Seton C, Waters KA, Collins JJ. Exploratory study of sleeping patterns in children admitted to Hospital. Journal of Paedriatrics and Child Health. 2014; 50(8):632-38. 14. Péreza MAI, Rodríguez AD, Insuga VS, Carral JD, Martín VP, Solana LGG. Prevalence of sleep disorders in patients with neurofibromatosis type 1 . Neurología. 2015; 30(9):561-5.

15. Stuttard L, Beresford B, Clarke S, Beecham J, Curtis J. A preliminary investigation into the effectiveness of a group-delivered sleep management intervention for parents of children with intellectual disabilities. Journal of Intellectual Disabilities. 2015; 19(4):342-55. 16. Teng YT, Chiang LC, Lue KH, Chang SW, Wang L, Lee SP, et al. Poor sleep quality measured by polysomnography in non-obese asthmatic children with or without moderate to severe obstructive sleep apnea. Sleep medicine. 2014; 15(9):1062-7.

17. Ward TM, Sonney J, Ringold S, Stockfish S, Wallace CA, Landis CA. Sleep disturbances and behavior problems in children with and without arthritis. J. pediatr. nurs. 2014; 29(4):321-28.

18. Matthews EE, Neu M, King N. Sleep in mother and child dyads during treatment for pediatric acute lymphoblastic leukemia. Oncol. nurs. forum. 2014; 1(6):599-610.

19. Hinds PS, Hockenberry M, Zhang L, Razzouk BI, McCarthy K, Cremer $L$, et al. Nocturnal awakenings, sleep environment interruptions, and fatigue in hospitalized children with cancer. Oncol. nurs. forum. 2007; 34(2):393-402.

20.Zupanec S, Jones H, Stremler R. Sleep habits and fatigue of children receiving maintenance chemotherapy for ALL and their parents. J. pediatr. oncol. nurs. 2014; 27(4):217-28.

21. Nunes MDR, Jacob E, Adlard K, Secola R, Nascimento L. Fatigue and sleep experiences at home in children and adolescents with cancer. Oncol. nurs. forum. 2015; 42(5):498-506.

22. Jacob E, Miaskowski C, Savedra M, Beyer JE, Treadwell M, Styles L. Changes in sleep, food intake, and activity levels during acute painful episodes in children with sickle cell disease. J. pediatr. nurs. 2006; 21(1):23-34.

23. Jacob E, Hesselgrave J, Sambuco G, Hockenberry M. Variations in pain, sleep, and activity during hospitalization in children with cancer. J. pediatr. oncol. nurs. 2007; 24(4):208-19.

24. Linder LA, Christian BJ. Nighttime sleep disruptions, the hospital care environment, and symptoms in elementary school-age Children with cancer. Oncol. nurs. forum. 2013; 39(6): 553-61. 25. Graves JK, Jacob E. Pain, coping, and sleep in children and adolescents with sickle cell disease. J. child adolesc. psychiatr. nurs._2014; 27(3):109-20.

26. Ward TM, Yuwen W, Voss J, Foell D, Gohar F, Ringold S. Sleep fragmentation and biomarkers in juvenile idiopathic arthritis. Biol. res. nurs. 2016; 18(3):299-06.

27. Heger ER, Calamaro CJ, Bentley LM, Hurley KM, Wang Y, Black MM. Nighttime sleep duration and sleep behaviors among toddlers from low-income families: associations with obesogenic behaviors and obesity and the role of parenting. Childhood Obesity. 2016; 12(5):392-400.

28. Lourenção DCA, Tronchin DMR. Segurança do paciente no ambiente cirúrgico: Tradução e adaptação cultural de instrumento validado. Acta paul. enfem. (Online) 2016; 29(1):1-8.

29. Alexandre NMC, Coluci MZO. Validade de conteúdo nos processos de construção e adaptação de instrumentos de medidas. Ciênc. saúde coletiva. (Online).2011; 16(7):3061-8.

30.Salgado APA, Adirson DM, Cava AM, Camacho KG. O sono do recém-nascido em unidade de terapia intensiva neonatal. Rev. enferm. UERJ. 2011; 19(4):644-9.

31. Hysing M, Sivertsen B, Stormark KM, Elgen I, Lundervold AJ. Sleep in children with chronic illness, and the relation to emotional and behavioral problems-A population-based study. Journal of Pediatric Psychology. 2009; 34(6):665-70.

32. Ferreira RER, Silvares EFM, Pires MLN, Assumpção FB, Moura JCB. Sono e comportamento em crianças. Rev. psicol.: teoria e prática. 2016; 18(2):159-72.

33.Zeller B, Loge JH, Kanellopoulos, A, Hamre H, Wyller VB, Ruu E. Chronic fatigue in long-term survivors of childhood lymphomas and leukemia: persistence and associated clinical factors. J. pediatr. hematol. oncol. 2014; 36(6):438-44.

34.Daniel L, Kazak AE, Li Yimei, Hobbie W, Ginsberg J, Butler E, et al. Relationship between sleep problems and psychological outcomes in adolescent and young adult cancer urvivors and controls. Support care cancer. 2016; 24(2): 539-46.

35. Goes ACJ, Reis LAB, Silva MBG, Kahlow BS, Skare TL. Artrite reumatoide e qualidade do sono. Rev. bras. reumatol. (Online). 2017; 57(4):294-8.

36. Góes SM, Cieslak F, Stefanello JMF, Milano GE, Paiva E, Leite N. Sono não-reparador e comorbidades associadas em mulheres com fibromialgia. Fisioter mov. 2009; 22(3):323-33.

37. Halal CSE, Matijasevich A, Howe LD, Santos IS, Barros FC, Nunes $\mathrm{ML}$. Short sleep duration in the first years of life and obesity/overweight at age 4 years: a birth cohort study. J. pediatr. 2016; 168(3):99-103. 38. Oliveira VM, Brasil MR, Chumlhak Z, Cordel PT, Czuy GHB, Silva SR. Nível de aptidão física em escolares: influência do índice de massa corporal, sexo e quantidade de sono. Rev. Saúde Meio Ambient. 2017; 6(1):4-17. 